\title{
Equilibrium Swelling of Pigment Gallstones: Evidence for Network Polymer Structure
}

\author{
BRIAN E. BLACK, University of Michigan, Ann Arbor, Michigan \\ 48104*; STEPHEN H. CARR, Department of Materials Science and \\ Engineering and Materials Research Center, Northwestern University, \\ Evanston, Illinois $60201^{\dagger}$; and HIDEKI OHKUBO and J. DONALD \\ OSTROW, Department of Medicine, Northwestern University and \\ Veterans Administration Lakeside Medical Center, Chicago, Illinois
} 60611

\begin{abstract}
Synopsis
A major component of pigment gallstones (PS) is a black, insoluble substance. It has been suggested that this pigment material might be a highly crosslinked polymer, and if such were the case, it should imbibe solvent (swell) to the maximum permitted by the crosslinks of its macromolecular network. We measured the equilibrium amount, $q_{\text {eq }}$, by which pulverized, desiccated PS swells in different liquids, including isotonic aqueous buffers at $\mathrm{pH}$ values from 1.5-11.5. For ionic strengths $\geq 0.15$, the dependence of $q_{\text {eq }}$ on $\mathrm{pH}$ exhibits a broad titration curve with a midpoint near $\mathrm{pH} 7 . \quad q_{\text {eq }}$ was $<1.2$ in methanol, dimethylformamide, dimethylsulfoxide, and chloroform. The ir absorbance from vinyl groups in the black pigment was only one-eighth that of unconjugated bilirubin, the primary chemical building block of PS; this implicates vinyl groups in the formation of a polymer network. The rise in $q_{\text {eq }}$ with increasing $\mathrm{pH}$ suggests that the carboxyl groups are free to ionize and are therefore not involved in the covalent bonds that make the crosslinked polymer. A network polymer structure would account for the inability to dissolve PS in those solvents in which unconjugated bilirubin is soluble.
\end{abstract}

\section{INTRODUCTION}

Pigment gallstones are composed, in large part, of a black material that is probably some insoluble derivative of bilirubin, ${ }^{1-3}$ but they also contain other substances, principally calcium phosphate and carbonate. ${ }^{4}$ Even calculi composed largely of cholesterol commonly contain small pigment particles at their core, dispersed throughout their bulk, and/or as exterior coatings. ${ }^{5,6}$ Thus, black pigment material based on bilirubin is present in virtually all gallbladder calculi.

Although insolubility can arise from physical association of bilirubin molecules with other species, including proteins or metal cations, it is plausible that the insolubility of the black pigment material results from chemical reactions joining bilirubin molecules covalently with each other. Such a proposition for bilirubin polymerization has been advanced previ-

* Present address: University of Illinois, College of Medicine, Chicago, Illinois 60680.

+ To whom correspondence should be addressed. 
ously, ${ }^{7,8}$ though never supported by experimental evidence. Furthermore, bilirubin possesses two vinyl substituents of the kind known to be capable of forming long-chain macromolecules from synthetic organic substances. ${ }^{9}$ The carboxylic acid groups and imine moieties are also potential sites for reaction of bilirubin with other molecules, including other bilirubin molecules.

It is, therefore, possible that the insoluble black pigment is simply a derivative resulting from self-polymerization of bilirubin or its calcium salt, a proposition rendered more plausible by the presence of multiple sites on the molecule for possible polymerization to occur. Furthermore, most of the likely polymerization schemes would result in formation of polymer networks, as opposed to collections of linear-chain polymers. A polymer network results whenever individual molecules participate in the building of several chains at once, thus becoming junction points connecting the several chains. Network polymers are always insoluble, amorphous solids, and such is also the case with the black material from pigment gallstones. ${ }^{1,5,10}$

Network polymers always also exhibit physical swelling ${ }^{11}$ when exposed to liquids that would have the ability to dissolve linear chains built from the corresponding monomer. Polymer networks are incapable of molecular-level dispersion in solution because their crosslinked nature prevents individual chains from separating from each other when exposed to the solvent. Rather, a network polymer imbibes solvent progressively, for periods ranging from a few hours to a few weeks, until it eventually grows to some equilibrium steady-state volume, $v_{\text {eq. }}$. The ratio of this equilibrium (swollen) volume to the original volume of the network polymer, $v_{0}$, is defined as the equilibrium swelling ratio, $q_{\mathrm{eq}}$. Thus, $q_{\mathrm{eq}}=v_{\mathrm{eq}} / v_{0}$. For a given polymer exposed to some solvent, networks that contain fewer junction points will swell to a larger extent than those networks in which the junction points are more closely spaced along the lengths of the chains. Thus, $q_{\text {eq }}$ becomes a direct measure of how tightly the network is crosslinked. Moreover, comparison of the degree of swelling of one particular network in any of several solvents can provide insight concerning which groups in the molecule might have been converted to polymer crosslinks and which groups have remained free to interact with the solvent.

The present paper reports application of these principles to study the swelling properties of black pigment gallstones in both aqueous buffers and organic solvents. The result provides the first clear evidence for a network polymer structure in these stones, which can explain their resistance to dissolution in virtually all solvents.

\section{MATERIALS AND METHODS}

Characteristic 2-5-mm black pigment stones ${ }^{10}$ were obtained at cholecystectomy. The stones were rinsed thoroughly with distilled water, blotted dry on cotton gauze, and then stored in a vacuum desiccator con- 
taining calcium sulfate desiccant. Subsequent studies revealed that these stones still retained $10-12 \%$ bound water and between $4.6-7.2 \%$ methanol-extractable substances. For various studies, the calculi were pulverized in an agate mortar to yield a powder consisting of angularly shaped particles in the size range of $0.1-5 \mu \mathrm{m}$. To prepare black pigment, the powdered stones were extracted first with 5 wt \% tetrasodium EDTA in distilled water and then with acidified chloroform/methanol $(2: 1, v / v) .{ }^{10}$ Finally, the powder was dried in a vacuum desiccator for 1 week.

Equilibrium swelling ratios, $q_{\text {eq }}$, were determined as follows: $1-3 \mathrm{mg}$ of powdered material was transferred to $0.8 \times 68-\mathrm{mm}$ glass capillary tubes (KIMAX-51, Kimble Glass Co., Toledo, $\mathrm{OH}$ ) that had been heat-sealed at one end, and the powder was tapped to the bottom of the tube. After centrifugation for $30 \mathrm{~s}$ at $11,600 \times g$ in a microhematocrit centrifuge (MB Centrifuge, Damon-IEC Division, Needham Heights, MA), the tubes were removed and mounted on the stage of a traveling microscope to measure the height of the packed plug of pigment powder. Then, using a microsyringe (Microfilter \#710, Hamilton Co., Reno, NV), the rest of the tube was filled with the swelling solvent. A gentle vacuum was applied to the top of the glass capillary to aspirate all the air occupying the interstices of the powder plug, the vacuum removed, and the solvent forced by atmospheric pressure into contact with each of the particles in the plug. It is assumed that any voids formed in the powder by the crushing step represent a negligible volume fraction of the pigment stone material. After recentrifugation and remeasurement of the height (never found to be significantly different from the height before addition of buffer) of the pigment plug, the tubes were maintained vertically at room temperature in the dark, and the height of the pigment column was determined serially, without recentrifuging, over the following weeks. The height of the column will be accurately proportional to the volume of each of its component particles (and independent of their shapes or distribution of sizes) under the reasonable condition that no appreciable shifting or rearranging of particles occurs during the course of a swelling experiment. Each datum in Figs. 2 and 3 is the mean value obtained from three completely independent experiments. Error bars indicate two standard deviations, calculated for a given stone by pooling (thus, sample size, $n$, of the experiment was 27 ) the variances found at each abscissa value.

Aqueous buffers were prepared from deionized, distilled water to an ionic strength of $0.15 \mathrm{M}$, using sodium potassium tartrate, acetic acid, potassium acid phosphate, and sodium borate as buffers in their appropriate $\mathrm{pH}$ ranges. $\mathrm{pH}$ was measured with a $\mathrm{pH}$ meter (model LS-S-30005, SargentWelch Company, Skokie, IL). Swelling was also measured in chloroform, methanol, dimethyl sulfoxide, and dimethylformamide, each freshly distilled in glass before using.

The ir absorption spectrophotometry was performed with a Nicolet model 7199 Fourier transform ir spectrophotometer. Bilirubin, powdered stone, or pre-extracted black pigment, $0.3-0.7 \mathrm{mg}$, was pulverized with 99 
mg spectral grade $\mathrm{KBr}$, and a wafer was prepared in a stainless-steel die under pressure of $3600 \mathrm{~atm}$ for $60 \mathrm{~s}$. Using the $1625-\mathrm{cm}^{-1}$ band as a reference for internal thickness, the absorbance of the vinyl groups of the pigments was measured at $991 \mathrm{~cm}^{-1}$. Unconjugated bilirubin, highly pure, was obtained from British Drug House through Gaillard-Schlesinger Corp., New York, NY.

\section{RESULTS}

At all $\mathrm{pH}$ values studied (1.6-11.6), pigment stone material swelled in the aqueous buffer solutions. Although much of the swelling occurred within the first $15 \mathrm{~min}, v_{\text {eq }}$ was not attained for more than $80 \mathrm{~h}$ at $\mathrm{pH}$ values greater than 7.0 and required at least $2 \mathrm{~h}$ even at $\mathrm{pH} 1.6$ (Fig. 1). Both the magnitude of swelling and the time to attain equilibrium increased with increasing $\mathrm{pH}$ values. As seen in Fig. 2, $q_{\mathrm{eq}}$ was relatively constant at values of ionic strength at or above the physiological value of $0.15 \mathrm{M}$; but $q_{\text {eq }}$ increased steeply as ionic strength decreased below $0.15 M$. For the two pigment gallstones studied at ionic strength $0.15 M$, a plot of $q_{\text {eq }} \mathrm{vs} \mathrm{pH}$ gave a broad titration curve with a midpoint between $\mathrm{pH} 7$ and 8 (Fig. 3). Swelling was observed also in organic solvents, though to a much lesser extent. Average values for $q_{\text {eq }}$ of the material from stone I were: 1.04 in

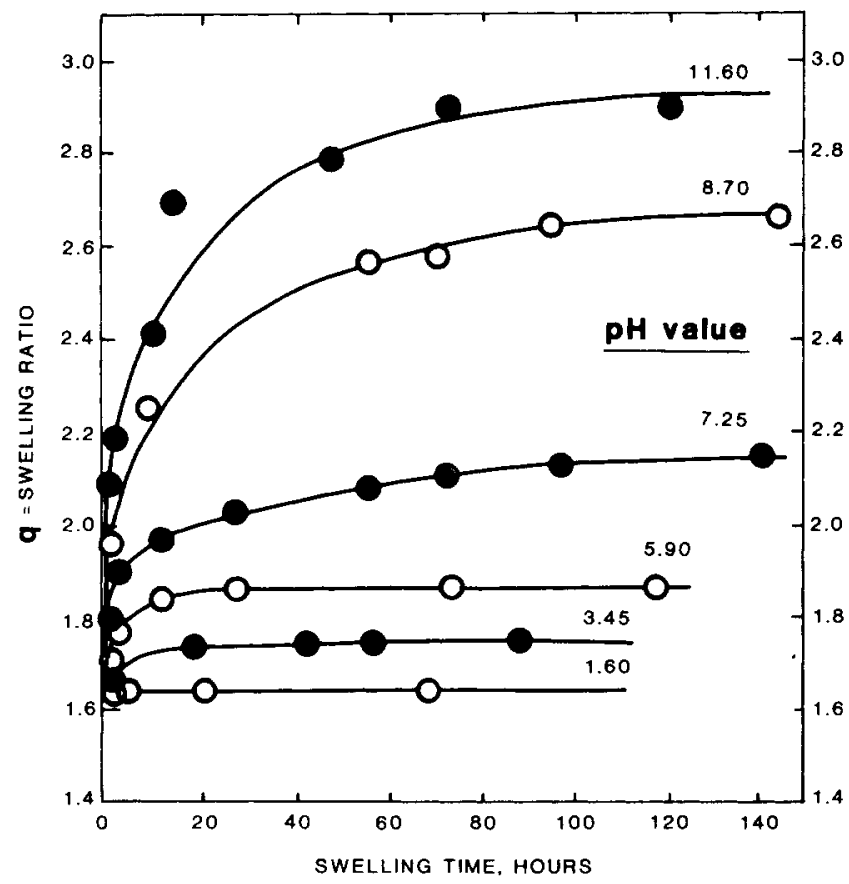

Fig. 1. Time-dependence of swelling of pigment gallstone material (patient 1) immersed in buffers of different $\mathrm{pH}$. Swelling ratio, $q$, is seen to rise until a steady level, $q_{\text {eq }}$, is reached. Ionic strength, $0.15 \mathrm{M}$. 


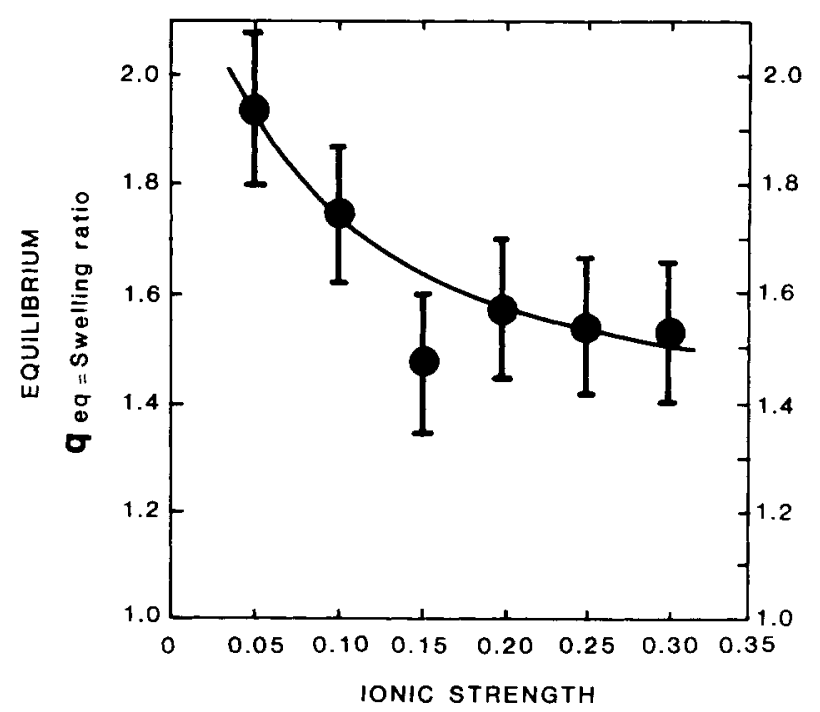

Fig. 2. Effect of ionic strength (at $p H$ 7) on equilibrium swelling ratio, $q_{\text {eq, }}$ of pigment gallstone material (patient 1). Ionic strength is in units of molarity.

dimethylformamide, 1.06 in dimethyl sulfoxide, 1.01 in methanol, and 1.19 in chloroform/methanol. The ir spectra obtained from pigment stone material (Fig. 4) and from pure bilirubin (Fig. 5) permit identification of different constituents that might be in these pigment stones; comparison of these two spectra also reveals changes in the bilirubin component of pigment stone material. Specifically, the relative ir absorbance at 991 $\mathrm{cm}^{-1}$, corresponding to the $\mathrm{C}-\mathrm{H}$ bond on the terminal vinyl groups, was 0.236 for unconjugated bilirubin but only 0.028 for extracted pigment gallstone material. One can see from these spectra that: (1) the bilirubin component of pigment stone material is present as a salt (probably the calcium salt; see Ref. 5); (2) there is no absorbance peak at $865 \mathrm{~cm}^{-1}$, which would indicate the presence of some calcium carbonate; and (3) there is little, if any, cholesterol. This latter point is based on the facts that cholesterol has strong absorbances in regions near 3450,2950 , and $1050 \mathrm{~cm}^{-1}$, and although the spectrum in Fig. 4 also shows absorbance in these same regions, this spectrum is essentially unchanged in these particular regions when it is obtained from this pigment material following prolonged extraction with chloroform/methanol.

\section{DISCUSSION}

Equilibrium swelling, ${ }^{11}$ with very low $q_{\text {eq }}$ values, suggests strongly that pigment gallstones contain a highly crosslinked polymer network. The most striking feature of this swelling was the marked dependence on $\mathrm{pH}$. Specifically, as $\mathrm{pH}$ was increased from 1.6 to 11.6 , the time to attain equilibrium was prolonged over 40 -fold and the $q_{\text {eq }}$ increased more than twofold. 


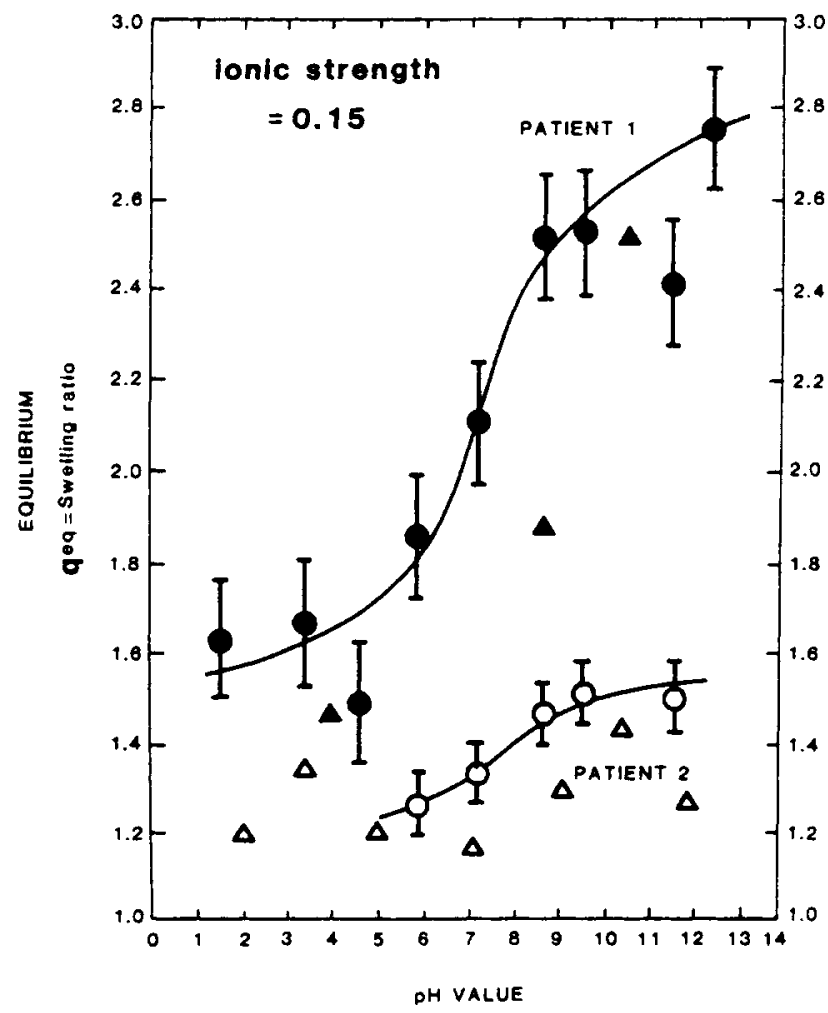

Fig. 3. $\mathrm{pH}$ dependence of equilibrium swelling ratio, $q_{\mathrm{eq}}$, for pigment gallstone material obtained from two different people. Ionic strength is $0.15 M$. Triangles indicate $q$ eq values for stone material that had not been extracted with the EDTA solution.

Most of the change in $q_{\text {eq }}$ occurred in the $\mathrm{pH}$ range of $4-10$, which is a broad range over which one may expect there to exist the $\mathrm{p} K_{a}$ values for the two carboxyl substituents on bilirubin. ${ }^{12,13}$ This suggests that some, or all, of these moieties may be free to interact with solvent, and thus they are presumably not involved in the covalent bonds that would link bilirubin molecules to build the polymer network.

This conclusion draws further support from the ir spectra, which revealed that the relative absorbance of the carboxyl groups in the black pigment is essentially the same as that in calcium bilirubinate. This indicates that these groups are not involved in covalent linkage in the polymer network. By contrast, absorbance of the pendant vinyl groups at $991 \mathrm{~cm}^{-1}$ was only one-eighth as intense in the pigment as in bilirubin. This implies that as much as seven-eights of the terminal vinyl groups of bilirubin may no longer be present in the pigment gallstone material, and thus most, if not all, are involved in the crosslinks of the network. Vinyl polymers are well known. ${ }^{14}$

Early work ${ }^{11}$ on the elasticity of crosslinked rubbers developed a quantitative relationship between $q_{\text {eq }}$ and $\bar{M}_{c}$, which is the average molecular 


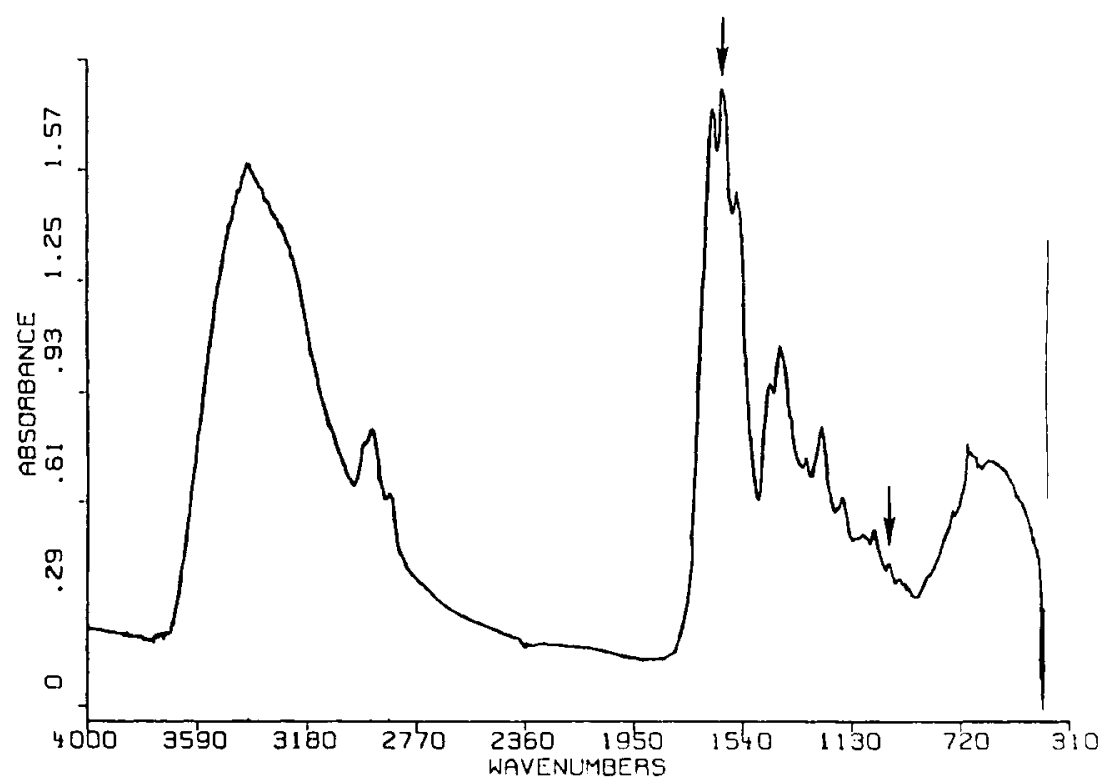

Fig. 4. The ir absorbance spectrum of pulverized pigment stone materials from patient 1. Arrows highlight the 1625 - and $991-\mathrm{cm}^{-1}$ bands.

weight of the chain segments joining junction points in the networks. A useful form of this relationship is

$$
\frac{1}{\bar{M}_{\mathrm{c}}}=\frac{2}{\bar{M}_{n}}-\frac{\left(\bar{v} / V_{1}\right)\left[\ln \left(1-q_{\mathrm{eq}}^{-1}\right)+q_{\mathrm{eq}}^{-1}+\bar{\chi}_{1} q_{\mathrm{eq}}^{-2}\right)}{v_{0}\left[q_{\mathrm{eq}}^{-1 / 3}-1 / 2\left(q_{\mathrm{eq}}^{-1}\right)\right]}
$$

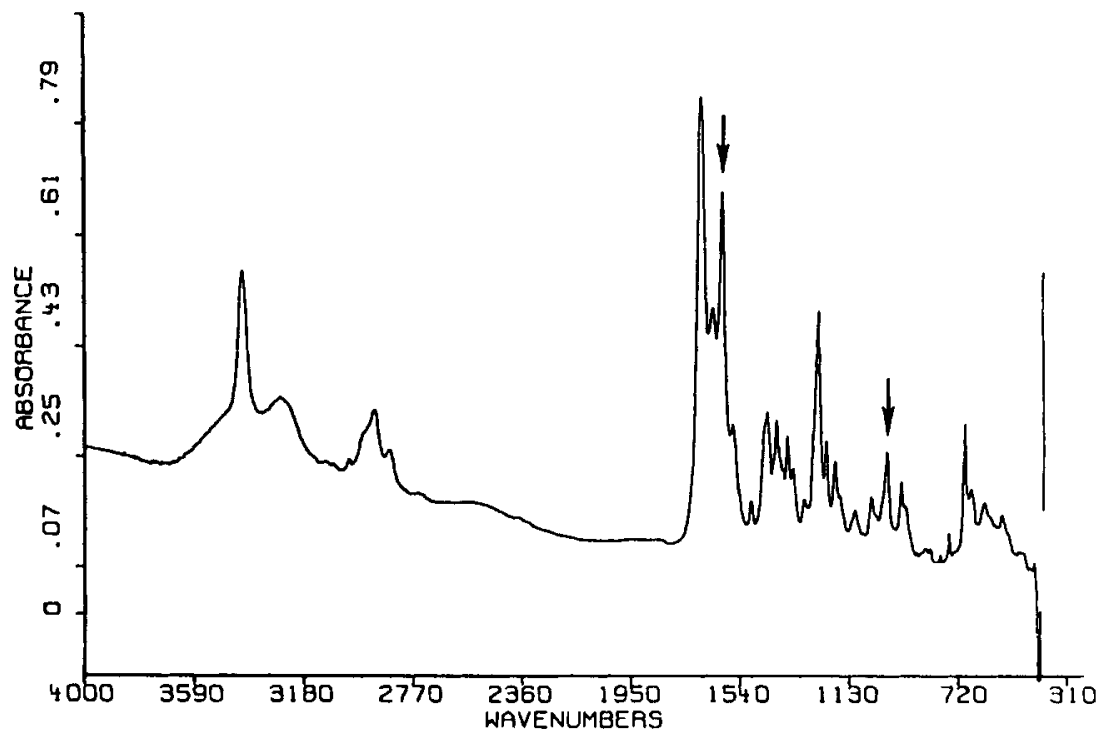

Fig. 5. The ir absorbance spectrum of powdered pure bilirubin. Arrows highlight the 1625and $991-\mathrm{cm}^{-1}$ bands. 
In this equation, $V_{1}$ is the molar volume of the solvent, $\bar{v}$ is the specific volume of the polymer, $\bar{M}_{n}$ is a characteristic molecular weight (taken in rubber elasticity theory as the number-average molecular weight of chains used initially to build the crosslinked network), and $\chi_{1}$ is the polymersolvent interaction parameter. $\chi_{1}$ ranges in value between zero and unity, and is a measure of how strongly the solvent interacts with the repeat units from which the polymer network is built. Strong solvents have high values of $\chi_{1}$ and correspondingly cause large swelling ratios to be observed.

The fact that $q_{\text {eq }}$ rises as the carboxyl groups become ionized may be explained by either or both the following propositions. The energetics of interaction between solvent (buffer) and polymer become more favorable as more parts of the molecule are ionized and, as a result, one can think of $\chi_{1}$ increasing. Alternatively, one might think of carboxyl groups at low $\mathrm{pH}$ forming effective crosslinks by participation in some hydrogen-bonding scheme with other parts of the network, and these noncovalent crosslinks are then lost as ionization of the carboxyl groups occurs. There is evidence in Fig. 3 in direct support of this point. Stone material that had not been extracted with EDTA solution prior to performing the equilibrium swelling experiment is seen (triangles) to swell to a lesser extent, especially in $\mathrm{pH}$ ranges above neutrality. (It is not clear whether this behavior applies to $q_{\text {eq values measured in acidic buffers.) This suggests that divalent metal }}$ ions, especially calcium, may play a role in stabilizing these ionic crosslinks. ${ }^{15}$ Loss of these "effective" crosslinks, as a result of metal ion extraction, will make the polymer network somewhat more loose and, therefore, able to swell by larger amounts.

The role of ionized moieties on the polymer is emphasized by the observed dependence on ionic strength in which higher ionic strengths lead to lower values of $q_{\text {eq. }}$. This effect could be thought of as more effective electrostatic shielding of ionized groups at higher ionic strength. Such a dependence on ionic strength, for material in which the network is the same for each datum ( $\bar{M}_{s}$ is the same), means that change in $q_{\text {eq }}$ with ionic strength reflect changes in $\chi_{1}$. Specifically, a decrease in swelling for some particular network would suggest that the strength of the solvent is correspondingly decreasing. Ionic strength would affect solvent "power" through its interactions with ionized moieties on the polymer network itself. It is unfortunate that one cannot obtain a precise value for $\chi_{1}$ for any of the buffer solutions used in these studies; typical values of $\chi_{1}$ were tried but were found to yield values of $\bar{M}_{c}$ that were smaller than even the formula weight of bilirubin. Thus, actual use of the equation yields an inconclusive result, but it suggests the notion that each bilirubin molecule in the pigment stone material serves as a junction point in the macromolecular network.

Average values of $q_{\text {eq }}$ were less than 1.2 with the four organic solvents tested. These values are much lower than those obtained with aqueous media, and they reflect the thermodynamically weak character of interaction these organic media have with the stone material. This observation 
emphasizes the fact that there are ionizable sites still on the network polymer, and they are the source of most of the polymer-solvent interaction that develops with aqueous (especially alkaline) media. The prospect that other non-bilirubin-derived constituents of pigment gallstones can participate in the swelling process cannot be overlooked. Specifically, the $\mathrm{pH}$ dependence discussed above might be influenced in some way by other electrolyte constituents. A greatly expanded study based on this work (pigment stones from $>60$ patients) is in progress and may be able to provide some resolution of this matter.

The degree of swelling and the effects of changes in $\mathrm{pH}$ values on $q_{\text {eq }}$ were much more striking with the stones from patient 1 as compared with patient 2. This suggests that $\bar{M}_{c}$ is larger in the stones from patient 1 , reflecting a lesser degree of crosslinking. However, the fact that stones from both patients exhibited similar functional relationships between $q_{\text {eq }}$ and $\mathrm{pH}$ values suggests that the character of the network was similar in both groups of stones. In our study in progress, we are measuring these swelling parameters on a wide variety of pigment stones and have determined that these characteristics do apply generally to all such stones, reflecting a common polymer structure, differing mainly in the degree of polymerization of the bilirubin component found in each stone.

In summary, equilibrium swelling with very low $q_{\text {eq }}$ values suggests strongly that pigment gallstones contain a polymer network. Lesser swelling in organic solvents, the rise in $q_{\text {eq }}$ with increasing $\mathrm{pH}$ values, and the dependence of $q_{\text {eq }}$ on ionic strength, all suggest that the ionizable carboxyl side chains are not involved in the covalent bonds that crosslink the bilirubin molecules within the polymer. The ir spectra support this conclusion and suggest further that the pendant vinyl groups are involved. Such a network polymer structure could account for the inability to dissolve pigment gallstones in solvents in which unconjugated bilirubin and/or its calcium salt are soluble.

This work was supported by a Veterans Administration Merit Review Research Grant and with funds from the Otho Sprague Foundation at Northwestern University Medical School. The technical assistance with ir spectroscopy by Ms. Kathleen A. Thomson is deeply appreciated. Use of central facilities of the Northwestern Materials Research Center, which is supported by National Science Foundation Grant DMR 79-23573, is gratefully acknowledged.

\section{References}

1. Soloway, R. D., Trotman, B. W. \& Ostrow, J. D. (1977) Gastroenterology 72, 167182.

2. Wosiewitz, U. \& Schroebler, S. (1978) Clin. Chim. Acta 89, 1-12.

3. Masuda, H. \& Nakayama, F. (1979) J. Lab. Clin. Med. 93, 353-360.

4. Sutor, D. J. \& Percival, J. M. (1978) Clir. Chim. Acta 89, 479-484.

5. Trotman, B. W., Morris, T. A., Sanchez, H. M., Soloway, R. D. \& Ostrow, J. D. (1977) Gastroenterology 72, 495-498.

6. Been, J. M., Bills, P. M. \& Lewis, D. (1979) Gastroenterology 76, 548-555.

7. Wosiewitz, U. \& Schroebler, S. (1978) Naturwissenschaften 65, 162-163.

8. Suzuki, N. (1965) Tohoku J. Exp. Med. 85, 396-405. 
9. Odian, G., (1970) Principles of Polymerization, McGraw-Hill, New York, Chap. 3.

10. Trotman, B. W., Ostrow, J. D. \& Soloway, R. D. (1974) Am. J. Digest. Dis. 19, 585590.

11. Flory, P. J. (1953) Principles of Polymer Chemistry, Cornell, Ithaca, N.Y., pp. 576589.

12. Overbeek, J. Th. G., Vink, C. L. J. \& Deesntra, H. (1955) Rec. Trav. Chim., Pays-Bas 74, 81-84.

13. Hansen, P. E., Thiessen, H. \& Brodersen, R. (1979) Acta Chem. Scand. B 33, $281-$ 293.

14. B. Vollmert (1973) Polymer Chemistry, Springer-Verlag, New York, pp. 33-205.

15. Eisenberg, A. \& King, A. M. (1977) Ion-Containing Polymers; Physical Properties and Structure, Academic Press, New York, Chap. 2.

Received June 8, 1981

Accepted August 25, 1981 